De Tampere à Séville : bilan de la sécurité européenne $(1 / 2)$

\title{
Version consolidée du Traité sur l'Union européenne (extrait)
}

Union européenne

\section{(2) OpenEdition}

\section{Journals}

Édition électronique

URL : http://journals.openedition.org/conflits/787

DOI : $10.4000 /$ conflits. 787

ISSN : $1777-5345$

Éditeur :

CCLS - Centre d'études sur les conflits lilberté et sécurité, L'Harmattan

Édition imprimée

Date de publication : 1 mars 2002

ISBN : 2-7475-3029-9

ISSN : 1157-996X

\section{Référence électronique}

Union européenne, «Version consolidée du Traité sur l"Union européenne (extrait) 》, Cultures \& Conflits [En ligne], 45 | printemps 2002, mis en ligne le 22 mars 2006, consulté le 30 mars 2021. URL : http:// journals.openedition.org/conflits/787 ; DOI : https://doi.org/10.4000/conflits.787

Ce document a été généré automatiquement le 30 mars 2021

Creative Commons License 


\title{
Version consolidée du Traité sur l'Union européenne (extrait)
}

\author{
Union européenne
}

\section{TITRE VI}

DISPOSITIONS RELATIVES À LA COOPÉRATION POLICIÈRE ET JUDICIAIRE EN MATIÈRE PÉNALE

Article 29(ex-article K.1)

Sans préjudice des compétences de la Communauté européenne, l'objectif de l'Union est d'offrir aux citoyens un niveau élevé de protection dans un espace de liberté, de sécurité et de justice, en élaborant une action en commun entre les Etats membres dans le domaine de la coopération policière et judiciaire en matière pénale, en prévenant le racisme et la xénophobie et en luttant contre ces phénomènes.

Cet objectif est atteint par la prévention de la criminalité, organisée ou autre, et la lutte contre ce phénomène, notamment le terrorisme, la traite d'êtres humains et les crimes contre des enfants, le trafic de drogue, le trafic d'armes, la corruption et la fraude, grâce :

- àune coopération plus étroite entre les forces de police, les autorités douanières et les autres autorités compétentes dans les Etats membres, àla fois directement et par l'intermé-diaire de l'office européen de police (Europol), conformément aux articles 30 et 32 ;

- à une coopération plus étroite entre les autorités judiciaires et autres autorités compétentes des Etats membres, conformément à l'article 31, points a) à d), et à l'article 32 ;

- au rapprochement, en tant que de besoin, des règles de droit pénal des Etats membres, conformément à l'article 31, pointe).

Article 30(ex-article K.2) 
1. L'action en commun dans le domaine de la coopération policière couvre entre autres :

a) la coopération opérationnelle entre les autorités compétentes, y compris les services de police, les services des douanes et autres services répressifs spécialisés des Etats membres, dans le domaine de la prévention et de la détection des infractions pénales et des enquêtes en la matière ;

b) la collecte, le stockage, le traitement, l'analyse et l'échange d'informations pertinentes, y compris d'informations détenues par des services répressifs concernant des signalements de transactions financières douteuses, notamment par l'intermédiaire d'Europol, sous réserve des dispositions appropriées relatives à la protection des données à caractère personnel ;

c) la coopération et les initiatives conjointes dans les domaines de la formation, des échanges d'officiers de liaison, des détachements, de l'utilisation des équipements et de la recherche en criminalistique ;

d) l'évaluation en commun de techniques d'enquête particulières concernant la détection des formes graves de criminalité organisée.

2. Le Conseil encourage la coopération par l'intermédiaire d'Europol et, en particulier, dans les cinq ans qui suivent la date d'entrée en vigueur du traité d'Amsterdam :

a) permet à Europol de faciliter et d'appuyer la préparation, et d'encourager la coordination et la mise en œuvre d'actions spécifiques d'enquête menées par les autorités compétentes des Etats membres, y compris des actions opérationnelles d'équipes conjointes, comprenant des représentants d'Europol à titre d'appui ;

b) arrête des mesures destinées à permettre à Europol de demander aux autorités compétentes des Etats membres de mener et de coordonner leurs enquêtes dans des affaires précises, et de développer des compétences spécialisées pouvant être mises à la disposition des Etats membres pour les aider dans des enquêtes sur la criminalité organisée ;

c) favorise l'établissement de contacts entre magistrats et enquêteurs spécialisés dans la lutte contre la criminalité organisée et travaillant en étroite coopération avec Europol ;

d) instaure un réseau de recherche, de documentation et de statistiques sur la criminalité transfrontière.

Article 31(ex-article K.3)

L'action en commun dans le domaine de la coopération judiciaire en matière pénale vise entre autres à

a) faciliter et accélérer la coopération entre les ministères et les autorités judiciaires ou équiva-lentes compétents des Etats membres pour ce qui est de la procédure et de l'exécution des décisions ;

b) faciliter l'extradition entre Etats membres ;

c) assurer, dans la mesure nécessaire à l'amélioration de cette coopération, la compatibilité des règles applicables dans les Etats membres ;

d) prévenir les conflits de compétences entre Etats membres ; 
e) adopter progressivement des mesures instaurant des règles minimales relatives aux éléments constitutifs des infractions pénales et aux sanctions applicables dans les domaines de la criminalité organisée, du terrorisme et du trafic de drogue.

Article 32(ex-article K.4)

Le Conseil fixe les conditions et les limites dans lesquelles les autorités compétentes visées aux articles 30 et 31 peuvent intervenir sur le territoire d'un autre Etat membre en liaison et en accord avec les autorités de celui-ci.

Article 33(ex-article K.5)

Le présent titre ne porte pas atteinte à l'exercice des responsabilités qui incombent aux Etats membres pour le maintien de l'ordre public et la sauvegarde de la sécurité intérieure.

Article 34(ex-article K.6)

1. Dans les domaines visés au présent titre, les Etats membres s'informent et se consultent mutuellement au sein du Conseil en vue de coordonner leur action. Ils instituent à cet effet une collaboration entre les services compétents de leurs administrations.

2. Le Conseil, sous la forme et selon les procédures appropriées indiquées dans le présent titre, prend des mesures et favorise la coopération en vue de contribuer à la poursuite des objectifs de l'Union. À cet effet, il peut, statuant à l'unanimité à l'initiative de tout Etat membre ou de la Commission :

a) arrêter des positions communes définissant l'approche de l'Union sur une question déter-minée ;

b) arrêter des décisions-cadres aux fins du rapprochement des dispositions législatives et régle-mentaires des Etats membres. Les décisions-cadres lient les Etats membres quant au résultat à atteindre, tout en laissant aux instances nationales la compétence quant à la forme et aux moyens. Elles ne peuvent entraîner d'effet direct ;

c) arrêter des décisions à toute autre fin conforme aux objectifs du présent titre, à l'exclusion de tout rapprochement des dispositions législatives et réglementaires des Etats membres. Ces décisions sont obligatoires et ne peuvent entraîner d'effet direct; le Conseil, statuant à la majorité qualifiée, arrête les mesures nécessaires pour mettre en œuvre ces décisions au niveau de l'Union ;

d) établir des conventions dont il recommande l'adoption par les Etats membres selon leurs règles constitutionnelles respectives. Les Etats membres engagent les procédures applicables dans le délai fixé par le Conseil.

Sauf dispositions contraires y figurant, ces conventions, une fois qu'elles ont été adoptées par la moitié au moins des Etats membres, entrent en vigueur dans les Etats membres qui les ont adoptées. Les mesures d'application de ces conventions sont adoptées au sein du Conseil à la majorité des deux tiers des Parties Contractantes.

3. Pour les délibérations du Conseil qui requièrent une majorité qualifiée, les voix des membres sont affectées de la pondération prévue à l'article 205, paragraphe 2, du traité insti-tuant la Communauté européenne; les délibérations sont acquises si elles ont recueilli au moins soixante-deux voix, exprimant le vote favorable d'au moins dix membres.

4. Pour les questions de procédure, les délibérations du Conseil sont acquises à la majorité des membres qui le composent. 


\section{Article 35(ex-article K.7)}

1. La Cour de justice des Communautés européennes est compétente, sous réserve des conditions définies au présent article, pour statuer à titre préjudiciel sur la validité et l'inter-prétation des décisions-cadres et des décisions, sur l'interprétation des conventions établies en vertu du présent titre, ainsi que sur la validité et l'interprétation de leurs mesures d'application.

2. Tout Etat membre peut, par une déclaration faite au moment de la signature du traité d'Amsterdam, ou à tout autre moment postérieur à ladite signature, accepter la compétence de la Cour de justice pour statuer à titre préjudiciel dans les conditions définies au paragraphe 1 .

3. Un Etat membre qui fait une déclaration au titre du paragraphe 2 indique que :

a) soit toute juridiction de cet Etat dont les décisions ne sont pas susceptibles d'un recours juridictionnel de droit interne a la faculté de demander à la Cour de justice de statuer à titre préjudiciel sur une question soulevée dans une affaire pendante devant elle et portant sur la validité ou l'interprétation d'un acte visé au paragraphe 1, lorsqu'elle estime qu'une décision sur ce point est nécessaire pour rendre son jugement,

b) soit toute juridiction de cet Etat a la faculté de demander à la Cour de justice de statuer à titre préjudiciel sur une question soulevée dans une affaire pendante devant elle et portant sur la validité ou l'interprétation d'un acte visé au paragraphe 1, lorsqu'elle estime qu'une décision sur ce point est nécessaire pour rendre son jugement.

4. Tout Etat membre, qu'il ait ou non fait une déclaration au titre du paragraphe 2, a le droit de présenter à la Cour des mémoires ou observations écrites dans les affaires dont elle est saisie en vertu du paragraphe 1.

5. La Cour de justice n'est pas compétente pour vérifier la validité ou la proportionnalité d'opérations menées par la police ou d'autres services répressifs dans un Etat membre, ni pour statuer sur l'exercice des responsabilités qui incombent aux Etats membres pour le maintien de l'ordre public et la sauvegarde de la sécurité intérieure.

6. La Cour de justice est compétente pour, contrôler la légalité des décisions-cadres et des décisions lorsqu'un recours est formé par un Etat membre ou par la Commission pour incom-pétence, violation des formes substantielles, violation du présent traité ou de toute règle de droit relative à son application, ou détournement de pouvoir. Les recours prévus au présent paragraphe doivent être formés dans un délai de deux mois à compter de la publication de l'acte.

7. La Cour de justice est compétente pour statuer sur tout différend entre Etats membres concernant l'interprétation ou l'application des actes adoptés au titre de l'article 34 , para-graphe 2 , dès lors que ce différend n'a pu être réglé au sein du Conseil dans les six mois qui ont suivi la saisine de celui-ci par l'un de ses membres. La Cour est également compétente pour statuer sur tout différend entre Etats membres et la Commission concernant l'interpréta-tion ou l'application des conventions établies en vertu de l'article 34, paragraphe 2, point d).

Article 36(ex-article K.8)

1. Il est institué un comité de coordination composé de hauts fonctionnaires. En plus de son rôle de coordination, ce comité a pour mission : 
- de formuler des avis à l'intention du Conseil, soit à la requête de celui-ci, soit de sa propre initiative ;

- de contribuer, sans préjudice de l'article 207 du traité instituant la Communauté européenne, à la préparation des travaux du Conseil dans les domaines visés à l'article 29.

2. La Commission est pleinement associée aux travaux dans les domaines visés au présent titre.

Article 37(ex-article K.9)

Les Etats membres défendent les positions communes arrêtées conformément au présent titre dans les organisations internationales et lors des conférences internationales auxquelles ils participent.

Les articles 18 et 19 s'appliquent, le cas échéant, aux questions relevant du présent titre.

Article 38(ex-article K.10)

Les accords visés à l'article 24 peuvent couvrir des matières relevant du présent titre.

Article 39(ex-article K.11)

1. Avant d'adopter toute mesure visée à l'article 34, paragraphe 2, points b), c) et d), le Conseil consulte le Parlement européen. Celui-ci rend son avis dans un délai que le Conseil peut déterminer et qui ne peut être inférieur à trois mois. À défaut d'avis rendu dans ce délai, le Conseil peut statuer.

2. La présidence et la Commission informent régulièrement le Parlement européen des travaux menés dans les domaines relevant du présent titre.

3. Le Parlement européen peut adresser des questions ou formuler des recommandations à l'intention du Conseil. Il procède chaque année à un débat sur les progrès réalisés dans les domaines visés au présent titre.

Article 40(ex-article K.12)

1. Les Etats membres qui se proposent d'instaurer entre eux une coopération renforcée peuvent être autorisés, dans le respect des articles 43 et 44 , à recourir aux institutions, procé-dures et mécanismes prévus par les traités, à condition que la coopération envisagée :

a) respecte les compétences de la Communauté européenne, de même que les objectifs fixés par le présent titre ;

b) ait pour but de permettre à l'Union de devenir plus rapidement un espace de liberté, de sécurité et de justice.

2. L'autorisation prévue au paragraphe 1 est accordée par le Conseil statuant à la majorité qualifiée à la demande des Etats membres concernés, la Commission ayant été invitée à présenter son avis. La demande est également transmise au Parlement européen.

Si un membre du Conseil déclare que, pour des raisons de politique nationale importantes et qu'il expose, il a l'intention de s'opposer à l'octroi d'une autorisation décidée à la majorité qualifiée, il n'est pas procédé au vote. Le Conseil, statuant à la majorité qualifiée, peut demander que le Conseil européen soit saisi de la question en vue d'une décision à l'unanimité. 
Les voix des membres du Conseil sont affectées de la pondération prévue à l'article 205, para-graphe 2, du traité instituant la Communauté européenne. Pour être adoptées, les décisions doivent recueillir au moins soixante-deux voix, exprimant le vote favorable d'au moins dix membres.

3. Tout Etat membre qui souhaite participer à la coopération instaurée en vertu du présent article notifie son intention au Conseil et à la Commission, qui transmet au Conseil, dans un délai de trois mois à compter de la date de réception de la notification, un avis éventuellement assorti d'une recommandation relative à des dispositions particulières qu'elle peut juger néces-saires pour que l'Etat membre concerné participe à la coopération en question. Dans un délai de quatre mois à compter de la date de la notification, le Conseil statue sur la demande ainsi que sur d'éventuelles dispositions particulières qu'il peut juger nécessaires. La décision est réputée approuvée, à moins que le Conseil, statuant à la majorité qualifiée, ne décide de la tenir en suspens; dans ce cas, le Conseil indique les motifs de sa décision et fixe un délai pour son réexamen. Aux fins du présent paragraphe, le Conseil statue dans les conditions prévues à l'article 44 .

4. Les dispositions des articles 29 à 41 s'appliquent à la coopération renforcée prévue par le présent article, sauf dispositions contraires de ce dernier et des articles 43 et 44 .

Les dispositions du traité instituant la Communauté européenne concernant la compétence de la Cour de justice des Communautés européennes et l'exercice de cette compétence s'appli-quent aux paragraphes 1, 2 et 3 .

5. Le présent article n'affecte pas les dispositions du protocole intégrant l'acquis de Schengen dans le cadre de l'Union européenne.

Article 41(ex-article K.13)

1. Les articles 189, 190, 195, 196 à 199, 203, 204, 205 paragraphe 3, aux articles 206 à 209, 213 à 219,255 et 290 du traité instituant la Communauté européenne sont applicables aux dispositions relatives aux domaines visés au présent titre.

2. Les dépenses administratives entraînées pour les institutions par les dispositions relatives aux domaines visés au présent titre sont à la charge du budget des Communautés européennes.

3. Les dépenses opérationnelles entraînées par la mise en œuvre desdites dispositions sont également à la charge du budget des Communautés européennes, sauf si le Conseil, statuant à l'unanimité, en décide autrement. Quand une dépense n'est pas mise à la charge du budget des Communautés européennes, elle est à la charge des Etats membres selon la clé du produit national brut, à moins que le Conseil, statuant à l'unanimité, n'en décide autrement.

4. La procédure budgétaire fixée dans le traité instituant la Communauté européenne s'applique aux dépenses qui sont à la charge du budget des Communautés européennes.

Article 42(ex-article K.14)

Le Conseil, statuant à l'unanimité à l'initiative de la Commission ou d'un Etat membre, et après consultation du Parlement européen, peut décider que des actions dans les domaines visés à l'article 29 relèveront du titre IV du traité instituant la Communauté européenne et, en même temps, déterminer les conditions de vote qui s'y rattachent. Il recommande l'adoption de cette décision par les Etats membres conformément à leurs règles constitutionnelles respec-tives.

(...) 
INDEX

Mots-clés : droit européen, textes et documents officiels 